\title{
Symbolic Model Checking of Stutter-Invariant Properties Using Generalized Testing Automata ${ }^{\star}$
}

\author{
Ala Eddine Ben Salem ${ }^{1,2}$, Alexandre Duret-Lutz ${ }^{1}$, \\ Fabrice Kordon ${ }^{2}$, and Yann Thierry-Mieg ${ }^{2}$ \\ 1 LRDE, EPITA, Le Kremlin-Bicêtre, France \\ $\{a l a$, adl $\}$ lrde.epita.fr \\ 2 Sorbonne Universités, UPMC Univ. Paris 06, \\ CNRS UMR 7606, LIP6, F-75005, Paris, France \\ \{Fabrice.Kordon, Yann.Thierry-Mieg\}@lip6.fr
}

\begin{abstract}
In a previous work, we showed that a kind of $\omega$-automata known as Transition-based Generalized Testing Automata (TGTA) can outperform the Büchi automata traditionally used for explicit model checking when verifying stutter-invariant properties.

In this work, we investigate the use of these generalized testing automata to improve symbolic model checking of stutter-invariant LTL properties. We propose an efficient symbolic encoding of stuttering transitions in the product between a model and a TGTA. Saturation techniques available for decision diagrams then benefit from the presence of stuttering self-loops on all states of TGTA. Experimentation of this approach confirms that it outperforms the symbolic approach based on (transition-based) Generalized Büchi Automata.
\end{abstract}

\section{Introduction}

Model checking for Linear-time Temporal Logic (LTL) is usually based on converting the negation of the property to check into an $\omega$-automaton $\mathcal{B}$, composing that automaton with a model $\mathcal{M}$ given as a Kripke structure, and finally checking the language emptiness of the resulting product $\mathcal{B} \otimes \mathcal{M}[21]$.

One way to implement this procedure is the explicit approach where $\mathcal{B}$ and $\mathcal{M}$ are represented as explicit graphs. $\mathcal{B}$ is usually a Büchi automaton or a generalization using multiple acceptance sets. We use Transition-based Generalized Büchi Automata (TGBA) for their conciseness. When the property to verify is stutter-invariant [8], testing automata [13] should be preferred to Büchi automata. Instead of observing the values of state propositions in the system, testing automata observe the changes of these values, making them suitable to represent stutter-invariant properties. In previous work [1], we showed how to generalize testing automata using several acceptance sets, and allowing a more efficient emptiness check. Our comparison showed these Transition-based Generalized Testing Automata (TGTA) to be superior to TGBA for model-checking of stutter-invariant properties.

Another implementation of this procedure is the symbolic approach where the automata and their products are represented by means of decision diagrams (a concise way to represent large sets or relations) [3]. Symbolic encodings for generalized Büchi

\footnotetext{
* This work has been partially supported by the project ImpRo/ANR-2010-BLAN-0317.
} 
automata are pretty common [17]. With such encodings, we can compute, in one step, the sets of all direct successors (PostImage) or predecessors (PreImage) of any set of states. Using this technique, there have been a lot of propositions for symbolic emptiness-check algorithms [9, 19, 14]. These symbolic algorithms manipulate fixpoints on the transition relation which can be optimized using saturation techniques [4, 20].

However these approaches do not offer any reduction when verifying stutter-invariant properties. So far, and to the best of our knowledge, testing automata have never been used in symbolic model checking. Our goal is therefore to propose a symbolic approach for model checking using TGTA, and compare it to the symbolic approach using TGBA. In particular, we show that the computation of fixpoints on the transition relation of the product can be sped up with a dedicated evaluation of stuttering transitions. We exploit a separation of the transition relation into two terms, one of which greatly benefits from saturation techniques.

This paper is organized as follows. Section 2 presents the symbolic model-checking approach for TGBA. For generality we define our symbolic structures using predicates over state variables in order to remain independent of the decision diagrams used to actually implement the approach. Section 3 focuses on the encoding of TGTA in the same framework. We first show how a TGTA can be encoded, then we show how to improve the encoding of the Kripke structure and the product to benefit from saturation in the encoding of stuttering transitions in the TGTA. Finally, Section 4 compares the two approaches experimentally with an implementation that uses hierarchical Set Decision Diagrams (SDD) [20] (a particular type of Decision Diagrams on integer variables, on which we can apply user-defined operations). On our large, BEEM-based benchmark, our symbolic encoding of TGTA appears to to be superior to TGBA.

\section{Symbolic LTL Model Checking Using TGBA}

We first recall how to perform the automata-theoretic approach to LTL model checking using symbolic encodings of TGBA and Kripke structures. This setup will serve as a baseline to measure our improvements from later sections.

Through the paper, let $A P$ designate the finite set of atomic propositions of the model. Any state of the model is labeled by a valuation of these atomic propositions. Let $\Sigma=2^{A P}$ denote the set of these valuations, which we interpret either as sets or as Boolean conjunctions. For instance if $A P=\{a, b\}$, then $\Sigma=2^{A P}=\{\{a, b\},\{a\},\{b\}, \emptyset\}$ or equivalently $\Sigma=\{a b, a \bar{b}, \bar{a} b, \bar{a} \bar{b}\}$. An execution of the model is an infinite sequence of such valuations, i.e., an element of $\Sigma^{\omega}$.

\subsection{Kripke Structures and Their Symbolic Encoding}

The executions of the model can be represented by a Kripke structure $\mathcal{M}$.

Definition 1 (Kripke Structure). A Kripke structure over $\Sigma$ is a tuple $\mathscr{M}=\left\langle S, S_{0}, R, L\right\rangle$, where:

- $S$ is a finite set of states,

$-S_{0} \subseteq S$ is the set of initial states,

$-R \subseteq S \times S$ is the transition relation,

- $L: S \rightarrow \Sigma$ is a state-labeling function. 
An execution $w=\ell_{0} \ell_{1} \ell_{2} \ldots \in \Sigma^{\omega}$ is accepted by $\mathcal{M}$ if there exists an infinite sequence $s_{0}, s_{1}, \ldots \in S^{\omega}$ such that $s_{0} \in S_{0}$ and $\forall i \in \mathbb{N},\left(L\left(s_{i}\right)=\ell_{i}\right) \wedge\left(\left(s_{i}, s_{i+1}\right) \in R\right)$. The language accepted by $\mathcal{M}$ is the set $\mathscr{L}(\mathscr{M}) \subseteq \Sigma^{\omega}$ of executions it accepts.

In symbolic model checking we encode such a structure with predicates that represent sets of states or transitions [18]. These predicates are then implemented using decision diagrams [3].

Definition 2 (Symbolic Kripke Structure). A Kripke structure $\mathcal{M}=\left\langle S, S_{0}, R, L\right\rangle$ can be encoded by the following predicates where $s, s^{\prime} \in S$ and $\ell \in \Sigma$ :

- $P_{S_{0}}(s)$ is true iff $s \in S_{0}$,

- $P_{R}\left(s, s^{\prime}\right)$ is true iff $\left(s, s^{\prime}\right) \in R$,

- $P_{L}(s, \ell)$ is true iff $L(s)=\ell$.

In the sequel, we use the notations $S_{0}(s), R\left(s, s^{\prime}\right)$ and $L(s, \ell)$ instead of $P_{S_{0}}(s), P_{R}\left(s, s^{\prime}\right)$ and $P_{L}(s, \ell)$. A Symbolic Kripke structure is therefore a triplet of predicates $K=\left\langle S_{0}, R, L\right\rangle$ on state variables.

Variables $s$ and $s^{\prime}$ used above are typically implemented using decision diagrams to represent either a state or a set of states. In a typical encoding [3], states are represented by conjunctions of Boolean variables. For instance if $S=\{0,1\}^{3}$, a state $s=(1,0,1)$ would be encoded as $s_{1} \bar{s}_{2} s_{3}$. Similarly, $s_{1} s_{3}$ would encode the set of states $\{(1,0,1),(1,1,1)\}$. With this encoding, $S_{0}, R$ and $L$ are propositional formulae which can be implemented with BDDs or other kind of decision diagrams. In our implementation, we used SDDs on integer variables [20].

\subsection{TGBA and Their Symbolic Encoding}

Transition-based Generalized Büchi Automata (TGBA) [11] are a generalization of the Büchi Automata (BA) commonly used for model checking. In our context, the TGBA represents the negation of the LTL property to verify. We chose to use TGBA rather than BA since they allow a more compact representation of properties [7].

Definition 3 (TGBA). $A$ TGBA over the alphabet $\Sigma=2^{A P}$ is a tuple $\mathcal{B}=\left\langle Q, Q_{0}, \delta, F\right\rangle$ where:

- $Q$ is a finite set of states,

- $Q_{0} \subseteq Q$ is a set of initial states,

- $\delta \subseteq Q \times \Sigma \times Q$ is a transition relation, where each element $\left(q, \ell, q^{\prime}\right)$ represents a transition from state $q$ to state $q^{\prime}$ labeled by the valuation $\ell$,

- $F \subseteq 2^{\delta}$ is a set of acceptance sets of transitions.

$\mathcal{B}$ accepts an execution $\ell_{0} \ell_{1} \ldots \in \Sigma^{\omega}$ if there exists an infinite path $\left(q_{0}, \ell_{0}, q_{1}\right)\left(q_{1}, \ell_{1}, q_{2}\right)$ $\ldots \in \delta^{\omega}$ that visits each acceptance set infinitely often: $q_{0} \in Q_{0}$ and $\forall f \in F, \forall i \in$ $\mathbb{N}, \exists j \geq i,\left(q_{j}, \ell_{j}, q_{j+1}\right) \in f$.

The language accepted by $\mathcal{B}$ is the set $\mathscr{L}(\mathcal{B}) \subseteq \Sigma^{\omega}$ of the executions it accepts.

We target TGBA in this paper because their use of generalized and transition-based acceptance make them more concise than traditional Büchi automata [11]. Generalized acceptance is classically used in symbolic model checking [9] and using transitionbased acceptance is not a problem [17]. People working with (classical) Büchi automata 
can adjust to our definitions by "pushing" the acceptance of states to their outgoing transitions [7].

Any LTL formula $\varphi$ can be converted into a TGBA whose language is the set of executions that satisfy $\varphi$ [7]. Figure 1(a) shows a TGBA derived from the LTL formula $\mathrm{FG} a$. The Boolean expression over $A P=\{a\}$ that labels each transition represents the valuation of atomic propositions that hold in this transition (in this example, $\Sigma=\{a, \bar{a}\}$ ). Any infinite path in this example is accepted if it visits infinitely often the only acceptance set containing transition $(1, a, 1)$.

Like Kripke structures, TGBAs can be encoded by predicates [18] on state variables.

\section{Definition 4 (Symbolic TGBA)}

A TGBA $\left\langle Q, Q_{0}, \delta, F\right\rangle$ is symbolically encoded by a triplet of predicates $\left\langle Q_{0}, \Delta,\left\{\Delta_{f}\right\}_{f \in F}\right\rangle$ where:

- $Q_{0}(q)$ is true iff $q \in Q_{0}$,

- $\Delta\left(q, \ell, q^{\prime}\right)$ is true iff $\left(q, \ell, q^{\prime}\right) \in \delta$,

- $\forall f \in F, \Delta_{f}\left(q, \ell, q^{\prime}\right)$ is true iff $\left(q, \ell, q^{\prime}\right) \in f$.

\subsection{Symbolic Product of a TGBA with a Kripke Structure}

We now show how to build a synchronous product by composing the symbolic representations of a TGBA with that of a Kripke structure, inspired from Sebastian et al. [18].

Definition 5 (Symbolic Product for TGBA). Given a Symbolic Kripke structure $K=$ $\left\langle S_{0}, R, L\right\rangle$ and a Symbolic TGBA $A=\left\langle Q_{0}, \Delta,\left\{\Delta_{f}\right\}_{f \in F}\right\rangle$ sharing a set AP of atomic propositions, the Symbolic Product $K \otimes A=\left\langle P_{0}, T,\left\{T_{f}\right\}_{f \in F}\right\rangle$ is defined by the predicates $P_{0}, T$ and $T_{f}$ encoding respectively the set of initial states, the transition relation and the acceptance transitions of the product:

- $(s, q)$ denotes the state variables of the product (s for the Kripke structure and q for TGBA),

- $P_{0}(s, q)=S_{0}(s) \wedge Q_{0}(q)$,

- $T\left((s, q),\left(s^{\prime}, q^{\prime}\right)\right)=\exists \ell\left[R\left(s, s^{\prime}\right) \wedge L(s, \ell) \wedge \Delta\left(q, \ell, q^{\prime}\right)\right]$, where $\left(s^{\prime}, q^{\prime}\right)$ encodes the next state variables,

- $\forall f \in F, T_{f}\left((s, q),\left(s^{\prime}, q^{\prime}\right)\right)=\exists \ell\left[R\left(s, s^{\prime}\right) \wedge L(s, \ell) \wedge \Delta_{f}\left(q, \ell, q^{\prime}\right)\right]$.

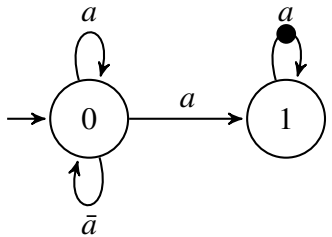

(a) TGBA

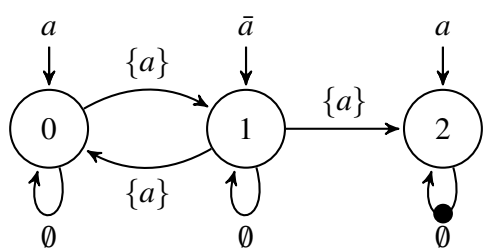

(b) TGTA

Fig. 1. TGBA and TGTA for the LTL property $\varphi=F G a$. Acceptance transitions are indicated by 
The labels $\ell$ are used to ensure that a transition $\left(q, \ell, q^{\prime}\right)$ of $A$ is synchronized with a state $s$ of $K$ such that $L(s, \ell)$. This way, we ensure that the product recognizes only the executions of $K$ that are also recognized by $A$. However we do not need to remember how product transitions are labeled to check $K \otimes A$ for emptiness. A product can be seen as a TGBA without labels on transitions.

In symbolic model checkers, the exploration of the product is based on the following PostImage operation [18]. For any set of states encoded by a predicate $P$, PostImage $(P)$ $\left(s^{\prime}, q^{\prime}\right)=\exists(s, q)\left[P(s, q) \wedge T\left((s, q),\left(s^{\prime}, q^{\prime}\right)\right)\right]$ returns a predicate representing the set of states reachable in one step a state in $P$.

Because in TGBA the acceptance conditions are based on transitions, we also define PostImage $(P, f)$ to computes the successors of $P$ reached using only transitions from an acceptance set $f \in F:$ PostImage $(P, f)\left(s^{\prime}, q^{\prime}\right)=\exists(s, q)\left[P(s, q) \wedge T_{f}\left((s, q),\left(s^{\prime}, q^{\prime}\right)\right)\right]$.

These two operations are at the heart of the symbolic emptiness check presented in the next section.

\subsection{Symbolic Emptiness Check}

One way to check if a product is not empty is to find a reachable Strongly Connected Component that contains transitions from all acceptance sets (we call it an accepting $S C C$ ). Figure 2 shows such an algorithm implemented using symbolic operations. It mimics the algorithm FEASIBLE of Kesten et al. [14] and can be seen as a forward variant of OWCTY (One Way Catch Them Young [9]) that uses PostImage computations instead of PreImage. Line 3 computes the set $P$ of all reachable states of the product. The main loop on lines 4-8 refines $P$ at each iteration. Lines 5-6 keep only the states of $P$ that can be reached from a cycle in $P$. Lines 7-8 then remove all cycles that never visit some acceptance set $f \in F$. Eventually the main loop will reach a fixpoint where $P$ contains all states that are reachable from an accepting SCC. The product is empty iff that set is empty.

There are many variants of such symbolic emptiness checks. We selected this variant mainly for its simplicity, as our contributions are mostly independent of the chosen algorithm: essentially, we will improve the cost of computing Reach $(P)$ (used lines 3 and 8).

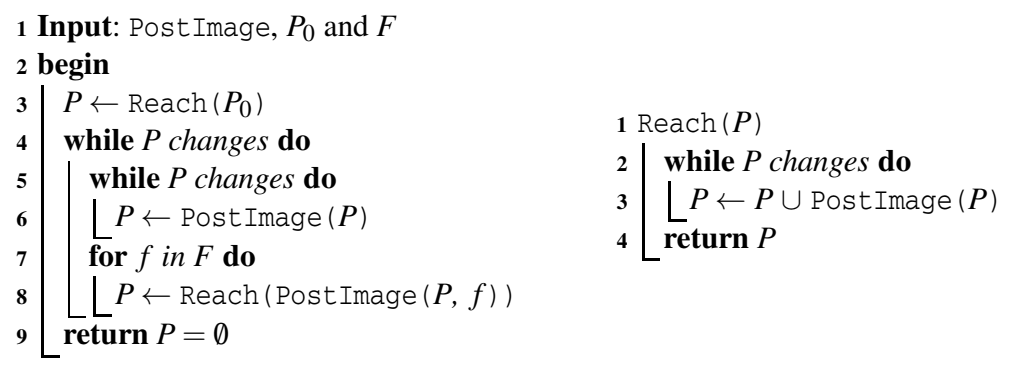

Fig. 2. Forward-variant of OWCTY, a symbolic emptiness check 


\section{Symbolic Approach Using TGTA}

Testing automata [10] are a kind of automata that recognize only stutter-invariant properties. In previous work [1] we generalized them as Transition-based Generalized Testing Automata (TGTA). In this section, we show how to encode a TGTA for symbolic model checking.

Definition 6. A property, i.e., a set of infinite sequences $\mathcal{P} \subseteq \Sigma^{\omega}$, is stutter-invariant iff any sequence $\ell_{0} \ell_{1} \ell_{2} \ldots \in \mathcal{P}$ remains in $\mathcal{P}$ after repeating any valuation $\ell_{i}$ or omitting duplicate valuations. Formally, $\mathcal{P}$ is stutter-invariant iff $\ell_{0} \ell_{1} \ell_{2} \ldots \in \mathcal{P} \Longleftrightarrow \ell_{0}^{i_{0}} \ell_{1}^{i_{1}} \ell_{2}^{i_{2}} \ldots \in$ $\mathcal{P}$ for any $i_{0}>0, i_{1}>0 \ldots$

Theorem 1. An LTL property is stutter-invariant iff it can be expressed as an LTL formula that does not use the $\mathrm{X}$ operator [16].

\subsection{Transition-Based Generalized Testing Automata}

While a TGBA observes the value of the atomic propositions AP, a TGTA observes the changes in these values. If a valuation of $A P$ does not change between two consecutive valuations of an execution, we say that a TGTA executes a stuttering transition.

If $A$ and $B$ are two valuations, $A \oplus B$ denotes the symmetric set difference, i.e., the set of atomic propositions that differ (e.g., $a \bar{b} \oplus a b=\{a\} \oplus\{a, b\}=\{b\}$ ). Technically, this can be implemented with an XOR operation on bitsets (hence the symbol $\oplus$ ).

Definition 7. A TGTA over the alphabet $\Sigma$ is a tuple $\mathcal{T}=\left\langle Q, Q_{0}, U, \delta, F\right\rangle$ where:

- $Q$ is a finite set of states,

- $Q_{0} \subseteq Q$ is a set of initial states,

- $U: Q_{0} \rightarrow 2^{\Sigma}$ is a function mapping each initial state to a set of symbols of $\Sigma$,

- $\delta \subseteq Q \times \Sigma \times Q$ is the transition relation, where each element $\left(q, c, q^{\prime}\right)$ represents a transition from state $q$ to state $q^{\prime}$ labeled by a changeset $c$ interpreted as a (possibly empty) set of atomic propositions whose value must change between $q$ and $q^{\prime}$,

- $F \subseteq 2^{\delta}$ is a set of acceptance sets of transitions,

and such that all stuttering transitions (i.e., transitions labeled by 0 ) are self-loops and every state has a stuttering self-loop. More formally, we can define a partition of $\delta=\delta_{\emptyset} \cup \delta_{*}$ where:

- $\delta_{\emptyset}=\{(q, \emptyset, q) \mid q \in Q\}$ is the stuttering transition relation,

- $\delta_{*}=\left\{\left(q, \ell, q^{\prime}\right) \in \delta \mid \ell \neq \emptyset\right\}$ is the non-stuttering transition relation.

An execution $\ell_{0} \ell_{1} \ell_{2} \ldots \in \Sigma^{\omega}$ is accepted by $\mathcal{T}$ if there exists an infinite path $\left(q_{0}, \ell_{0} \oplus\right.$ $\left.\ell_{1}, q_{1}\right)\left(q_{1}, \ell_{1} \oplus \ell_{2}, q_{2}\right)\left(q_{2}, \ell_{2} \oplus \ell_{3}, q_{3}\right) \ldots \in \delta^{\omega}$ where:

- $q_{0} \in Q_{0}$ with $\ell_{0} \in U\left(q_{0}\right)$ (the execution is recognized by the path),

- $\forall f \in F, \forall i \in \mathbb{N}, \exists j \geq i,\left(q_{j}, \ell_{j} \oplus \ell_{j+1}, q_{j+1}\right) \in f$ (each acceptance set is visited infinitely often).

The language accepted by $\mathcal{T}$ is the set $\mathscr{L}(\mathcal{T}) \subseteq \Sigma^{\omega}$ of executions it accepts.

Figure 1(b) shows a TGTA recognizing the LTL formula FGa. Acceptance sets are represented using dots as in TGBAs. Transitions are labeled by changesets: e.g., the transition $(0,\{a\}, 1)$ means that the value of $a$ changes between states 0 and 1 . Initial 
valuations are shown above initial arrows: $U(0)=\{a\}, U(1)=\{\bar{a}\}$ and $U(2)=\{a\}$. As an illustration, the execution $\bar{a} ; a ; a ; a ; \ldots$ is accepted by the run $(1) \stackrel{\{a\}}{\longrightarrow}(2) \stackrel{0}{\rightarrow}$ (2) $\stackrel{0}{\rightarrow}$ (2) ... because the value $a$ only changes between the first two steps.

Theorem 2. Any stutter-invariant property can be translated into an equivalent TGTA [1]].

Note that Def. 7] differs from our previous work [1] because we now enforce a partition of $\delta$ such that stuttering transitions can only be self-loops. However, the TGTA resulting from the LTL translation we presented previously [1] already have this property. We will use it to optimize symbolic computation in section 3.3

Finally, a TGTA's symbolic encoding is similar to that of a TGBA.

\section{Definition 8 (Symbolic TGTA)}

A TGTA $\mathcal{T}=\left\langle Q, Q_{0}, U, \delta, F\right\rangle$ is symbolically encoded by a triplet of predicates $\left\langle U_{0}, \Delta^{\oplus},\left\{\Delta_{f}^{\oplus}\right\}_{f \in F}\right\rangle$ where:

- $U_{0}(q, \ell)$ is true iff $\left(q \in Q_{0}\right) \wedge(U(q)=\ell)$

- $\Delta^{\oplus}\left(q, c, q^{\prime}\right)$ is true iff $\left(q, c, q^{\prime}\right) \in \delta$

- $\forall f \in F, \Delta_{f}^{\oplus}\left(q, c, q^{\prime}\right)$ is true iff $\left(\left(q, c, q^{\prime}\right) \in f\right)$

\subsection{Symbolic Product of a TGTA with a Kripke Structure}

The product between a TGTA and a Kripke structure is similar to the TGBA case, except that we have to deal with changesets. The transitions $\left(s, s^{\prime}\right)$ of a Kripke structure that must be synchronized with a transition $\left(q, c, q^{\prime}\right)$ of a TGTA, are all the transitions such that the label of $s$ and $s^{\prime}$ differs by the changeset $c$.

In order to reduce the number of symbolic operations when computing the Symbolic product of a TGTA with a Kripke structure, we introduce a changeset-based encoding of Kripke structure (only the transition relation changes).

Definition 9 (Changeset-based symbolic Kripke structure). A Kripke structure $\mathcal{M}=$ $\left\langle S, S_{0}, R, L\right\rangle$, can be encoded by the changeset-based symbolic Kripke structure $K^{\oplus}=$ $\left\langle S_{0}, R^{\oplus}, L\right\rangle$, where:

- the predicate $R^{\oplus}\left(s, c, s^{\prime}\right)$ is true iff $\left(\left(s, s^{\prime}\right) \in R \wedge\left(L(s) \oplus L\left(s^{\prime}\right)\right)=c\right)$,

- the predicates $S_{0}$ and $L$ have the same definition as for a Symbolic Kripke structure $K($ Def. 2).

In practice, the (changeset-based or not) symbolic transition relation of the Kripke structure should be constructed directly from the model and atomic propositions of the formula to check. In Section 4.2, we discuss how we build such changeset-based Kripke structures in our setup.

The procedure requires reconstruction of the symbolic transition relation for each formula (or at least for each set of atomic propositions used in the formulas). However the cost of this construction is not significant with respect to the complexity of the overall model checking procedure (overall on our benchmark, less than $0.16 \%$ of total time was spent building these transition relations).

Adjusting the symbolic encoding of the Kripke structure to TGTA, allows us to obtain the following natural definition of the symbolic product using TGTA: 
Definition 10 (Symbolic Product for TGTA). Given a changeset-based Symbolic Kripke structure $K^{\oplus}=\left\langle S_{0}, R, L\right\rangle$ and a Symbolic TGTA $A^{\oplus}=\left\langle U_{0}, \Delta^{\oplus},\left\{\Delta_{f}^{\oplus}\right\}_{f \in F}\right\rangle$ sharing the same set of atomic propositions AP, the Symbolic Product $K^{\oplus} \otimes A^{\oplus}=\left\langle P_{0}, T,\left\{T_{f}\right\}_{f \in F}\right\rangle$ is defined by the following predicates:

- The set of initial states is encoded by: $P_{0}(s, q)=\exists \ell\left[S_{0}(s) \wedge L(s, \ell) \wedge U_{0}(q, \ell)\right]$

- The transition relation of the product is: $T\left((s, q),\left(s^{\prime}, q^{\prime}\right)\right)=\exists c\left[R^{\oplus}\left(s, c, s^{\prime}\right) \wedge \Delta^{\oplus}\left(q, c, q^{\prime}\right)\right]$

- The definition of $T_{f}$ is similar to $T$ by replacing $\Delta^{\oplus}$ with $\Delta_{f}^{\oplus}$.

The definitions of PostImage $(P)$ and PostImage $(P, f)$ are the same as in the TGBA approach, with the new expressions of $T$ and $T_{f}$ above.

As for the product in TGBA approach, the product in TGTA approach is a TGBA (or a TGTA) without labels on transitions, and the same emptiness check algorithm (Fig.2) can be used for the two products.

\subsection{Exploiting Stuttering Transitions to Improve Saturation in the TGTA Approach}

Among symbolic approaches for evaluating a fixpoint on a transition relation, the saturation algorithm offers gains of one to three orders of magnitude [4] in both time and memory, especially when applied to asynchronous systems [5].

The saturation algorithm does not use a breadth-first exploration of the product (i.e., each iteration in the function Reach (Fig.2) is not a "global" PostImage() computation). Saturation instead recursively repeats "local" fixed-points by recognizing and exploiting transitions locality and identity transformations on state variables [5].

This algorithm considers that the system state consists of $k$ discrete variables encoded by a Decision Diagram, and that the transition relation is expressed as a disjunction of terms called transition clusters. Each cluster typically only reads or writes a limited subset consisting of $k^{\prime} \leq k$ variables, called the support of the cluster. During the least fixpoint computing the reachable states, saturation technique consists in reordering [12] the evaluation of ("local" fixed-points on) clusters in order to avoid the construction of (useless) intermediate Decision Diagram nodes.

The algorithm to determine an ordering for saturation is based on the support of each cluster.

We now show how to decompose the transition relation of the product $K^{\oplus} \otimes A^{\oplus}$ to exhibit clusters having a smaller support, favoring the saturation technique.

We base our decomposition on the fact that in a TGTA, all stuttering transitions are self-loops and every state has a stuttering self-loop $\left(\delta_{\emptyset}\right.$ in Def. 7 ). Therefore, stuttering transitions in the Kripke structure can be mapped to stuttering transitions in the product regardless of the TGTA state.

Let us separate stuttering and non-stuttering transitions in the transition relation $T$ of the product between a Kripke structure and a TGTA $\left(K^{\oplus} \otimes A^{\oplus}\right)$ :

$$
T\left((s, q),\left(s^{\prime}, q^{\prime}\right)\right)=\left(R^{\oplus}\left(s, \emptyset, s^{\prime}\right) \wedge \Delta^{\oplus}\left(q, \emptyset, q^{\prime}\right)\right) \vee\left(\exists c\left[R_{*}^{\oplus}\left(s, c, s^{\prime}\right) \wedge \Delta_{*}^{\oplus}\left(q, c, q^{\prime}\right)\right]\right)
$$

where $R_{*}^{\oplus}$ and $\Delta_{*}^{\oplus}$ encode respectively the non-stuttering transitions of the model and of the TGTA: 
- $\Delta_{*}^{\oplus}\left(q, c, q^{\prime}\right)$ is true iff $\left(q, c, q^{\prime}\right) \in \boldsymbol{\delta}_{*}$ (see Def.7)

- $R_{*}^{\oplus}\left(s, c, s^{\prime}\right)$ is true iff $R^{\oplus}\left(s, c, s^{\prime}\right) \wedge(c \neq \emptyset)$

According to the definition of $\delta_{\emptyset}$ in Def. 7 the predicate $\Delta^{\oplus}\left(q, \emptyset, q^{\prime}\right)$ encodes the set of TGTA's self-loops and can be replaced by the predicate equal $\left(q, q^{\prime}\right)$, simplifying $T$ :

$$
T\left((s, q),\left(s^{\prime}, q^{\prime}\right)\right)=(\underbrace{R^{\oplus}\left(s, \emptyset, s^{\prime}\right) \wedge \operatorname{equal}\left(q, q^{\prime}\right)}_{T_{\theta}\left((s, q),\left(s^{\prime}, q^{\prime}\right)\right)}) \vee(\underbrace{\exists c\left[R_{*}^{\oplus}\left(s, c, s^{\prime}\right) \wedge \Delta_{*}^{\oplus}\left(q, c, q^{\prime}\right)\right]}_{T_{*}\left((s, q),\left(s^{\prime}, q^{\prime}\right)\right)})
$$

The transition relation (1) is a disjunction of $T_{*}$, synchronizing updates of both TGTA and Kripke structure, and $T_{0}$, corresponding to the stuttering transitions of the Kripke structure. Since all states in the TGTA have a stuttering self-loop, $T_{\emptyset}$ does not depend on the TGTA state. In practice, the predicate equal $\left(q, q^{\prime}\right)$ is an identity relation for variable $q$ [5] and is simplified away (i.e., the term $T_{0}$ can be applied to a decision diagram without consulting or updating the variable $q[12])$. Hence $q$ is not part of the clusters supports in $T_{0}$ (while $q$ is part of the clusters supports in $T_{*}$ ). This gives more freedom to the saturation technique for reordering the application of clusters in $T_{0}$.

Note that in the product of TGBA with Kripke structure (Def. 5) there is no $T_{0}$ that could be extracted since there is no stuttering hypothesis in general. This severely limits the possibilities of the saturation algorithm in the TGBA approach.

In the symbolic emptiness check presented in Fig. 2, the function Reach corresponds to a least fixpoint performed using saturation. As we shall see experimentally in the next section, the better encoding of $T_{\emptyset}$ (without $q$ in its support) in the product of TGTA with Kripke structure, greatly favors the saturation technique, leading to gains of roughly one order of magnitude.

\section{Experimentation}

We now compare the approaches presented in this paper. The symbolic model-checking approach using TGBA, presented in Section 2 serves as our baseline. We first describe our implementation and selected benchmarks, prior to discussing the results.

\subsection{Implementation}

All approaches are implemented on top of three libraries: Spot, SDD/ITS, and LTSmin.

Spot is a model-checking library providing several bricks that can be combined to build model checkers [7]. In our implementation, we reused the modules providing a translation form an LTL formula into a TGBA and into a TGTA [1].

SDD/ITS is a library for symbolic representation of state spaces in the form of Instantiable Transition Systems (ITS): an abstract interface for symbolic Labeled Transition Systems (LTS). The symbolic encoding of ITS is based on Hierarchical Set Decision Diagrams (SDD) [20]. SDDs allow a compact symbolic representation of states and transition relation.

\footnotetext{
${ }^{1}$ Respectively http://spot.lip6.fr. http://ddd.lip6.fr. and http://fmt.cs.utwente.nl/tools/ltsmin.
} 
The algorithms presented in this paper can be implemented using any kind of decision diagram (such as OBDD), but use of the SDD software library allows to easily benefit from the automatic saturation mechanism described in [12].

LTSmin [2] can generate state spaces from various input formalisms ( $\mu$ CRL, DVE, GNA, MAPLE, PROMELA, ...) and store the obtained LTS in a concise symbolic format, called Extended Table Format (ETF). We used LTSmin to convert DVE models into ETF for our experiments. This approach offers good generality for our tool, since it can process any formalism supported by LTSmin tool.

Our symbolic model checker inputs an ETF file and an LTL formula. The LTL formula is converted into TGBA or TGTA which is then encoded using an ITS. The ETF model is also symbolically encoded using an ITS (see Sec.4.2). The two obtained ITSs are then composed to build a symbolic product, which is also an ITS. Finally, the OWCTY emptiness check is applied to this product.

\subsection{Using ETF to Build a Changeset-Based Symbolic Kripke Structure}

An ETF file 2 produced by LTSmin is a text-based serialization of the symbolic representation of the transition relation of a model whose states consist in $k$ integer variables. Transitions are described in the following tabular form:

$\begin{array}{llll}0 / 1 & 0 / 1 & * & * \\ 1 / 2 & * & 0 / 1 & * \\ \ldots & & & \end{array}$

where each column correspond to a variable, and each line describes the effect of a symbolic transition on the corresponding variables. The notation "in/out" means that the variable must have the value "in" for the transition to fire, and the value is then updated to "out". A “*” means that the variable is not consulted or updated by the transition. Each line may consequently encode a set of explicit transitions that differ only by the values of the starred variables: the support of a transition is the set of unstarred variables.

A changeset-based symbolic Kripke structure, as defined in Sec. 3.2 can be easily obtained from such a description. To obtain a changeset associated to a line in the file, it is enough to compute difference between values of atomic propositions associated to the in variables and the values associated to the out variables. Because they do not change, starred variables have no influence on the changeset.

Note that an empty changeset does not necessarily correspond to a line where all variables are starred. Even when in and out values are different, they may have no influence on the atomic propositions, and the resulting changeset may be empty. For instance if the only atomic proposition considered is $p=\left(v_{1}>1\right)$ (where $v_{1}$ denotes the first-column variable), then the changeset associated to the first line is $\emptyset$, and the changeset for the second line is $\{p\}$.

\subsection{Benchmark}

We evaluated the TGBA and TGTA approaches on the following models and formulae:

2 http://fmt.cs.utwente.nl/tools/ltsmin/doc/etf.html 
Table 1. Characteristics of our selected benchmark models. The stuttering-ratio represents the percentage of stuttering transitions in the model. Since the definition of stuttering depends on the atomic propositions of the formula, we give an average over the 200 properties checked against each model.

\begin{tabular}{|c|c|c|c|c|c|}
\hline BEEM model & $\begin{array}{l}\text { states } \\
10^{3} \times\end{array}$ & $\begin{array}{l}\text { stut. } \\
\text { ratio }\end{array}$ & BEEM model & $\begin{array}{l}\text { states } \\
10^{3} \times\end{array}$ & $\begin{array}{l}\text { stut. } \\
\text { ratio }\end{array}$ \\
\hline at. 5 & 31999 & $95 \%$ & lann. 6 & 144151 & $52 \%$ \\
\hline bakery. 4 & 157 & $83 \%$ & lann. 7 & 160025 & $64 \%$ \\
\hline bopdp. 3 & 1040 & $91 \%$ & lifts. 7 & 5126 & $93 \%$ \\
\hline elevator. 4 & 888 & $74 \%$ & peterson. 5 & 131064 & $83 \%$ \\
\hline brp2.3 & 40 & $79 \%$ & pgm_protocol.8 & 3069 & $92 \%$ \\
\hline fischer. 5 & 101028 & $89 \%$ & phils. 8 & 43046 & $89 \%$ \\
\hline lamport_nonatomic.5 & 95118 & $92 \%$ & production_cell.6 & 14520 & $85 \%$ \\
\hline lamport. 7 & 38717 & $93 \%$ & reader_writer.3 & 604 & $88 \%$ \\
\hline
\end{tabular}

- Our models come from the BEEM benchmark [15], a suite of models for explicit model checking, which contains some models that are considered difficult for symbolic model checkers [2]. Table 1] summarizes the 16 models we selected as representatives of the overall benchmark.

- BEEM provides a few LTL formulae, but they mostly represent safety properties and can thus be checked without building a product. Therefore, for each model, we randomly generated 200 stutter-invariant LTL formulae: 100 verified formulae (empty product) and 100 violated formulae (non-empty product). We consequently have a total 3200 pairs of (model, formula).

All tests were run on a 64bit Linux system running on an Intel Xeon E5645 at 2.40GHz. Executions that exceeded 30 minutes or $4 \mathrm{~GB}$ of RAM were aborted and are reported with time and memory above these thresholds in our graphics.

In all approaches evaluated, symbolic products are encoded using the same variable ordering: we used the symbolic encoding named "log-encode with top-order" by Sebastiani et al. [18].

\subsection{Results}

The results of our experimenta 3 comparisons are presented by the two scatter plot matrices of Fig. 3 and Fig. 4. The scatter plot highlighted at the bottom of Fig. 3 compares the time-performance of the TGTA-approach against the reference TGBA approach 4 Each point of the scatter plot represents a measurement for a pair (model,formula). For the highlighted plot, the $\mathrm{x}$-axis represents the TGBA approach and the y-axis represent the TGTA approach, so 3060 points below the diagonal correspond to cases where the TGTA approach is better, and the 131 points above the diagonal corresponds to points were the TGBA approach is better (In scatter plot matrices, each point below the diagonal is in favor of the approach displayed on the right, while each point above the

\footnotetext{
${ }^{3}$ The results, models, formulae and tools used in these tests, can be downloaded from http://www.lrde.epita.fr/ ala/TACAS-2014/Benchmark.html

${ }^{4}$ We recommend viewing these plots online.
} 


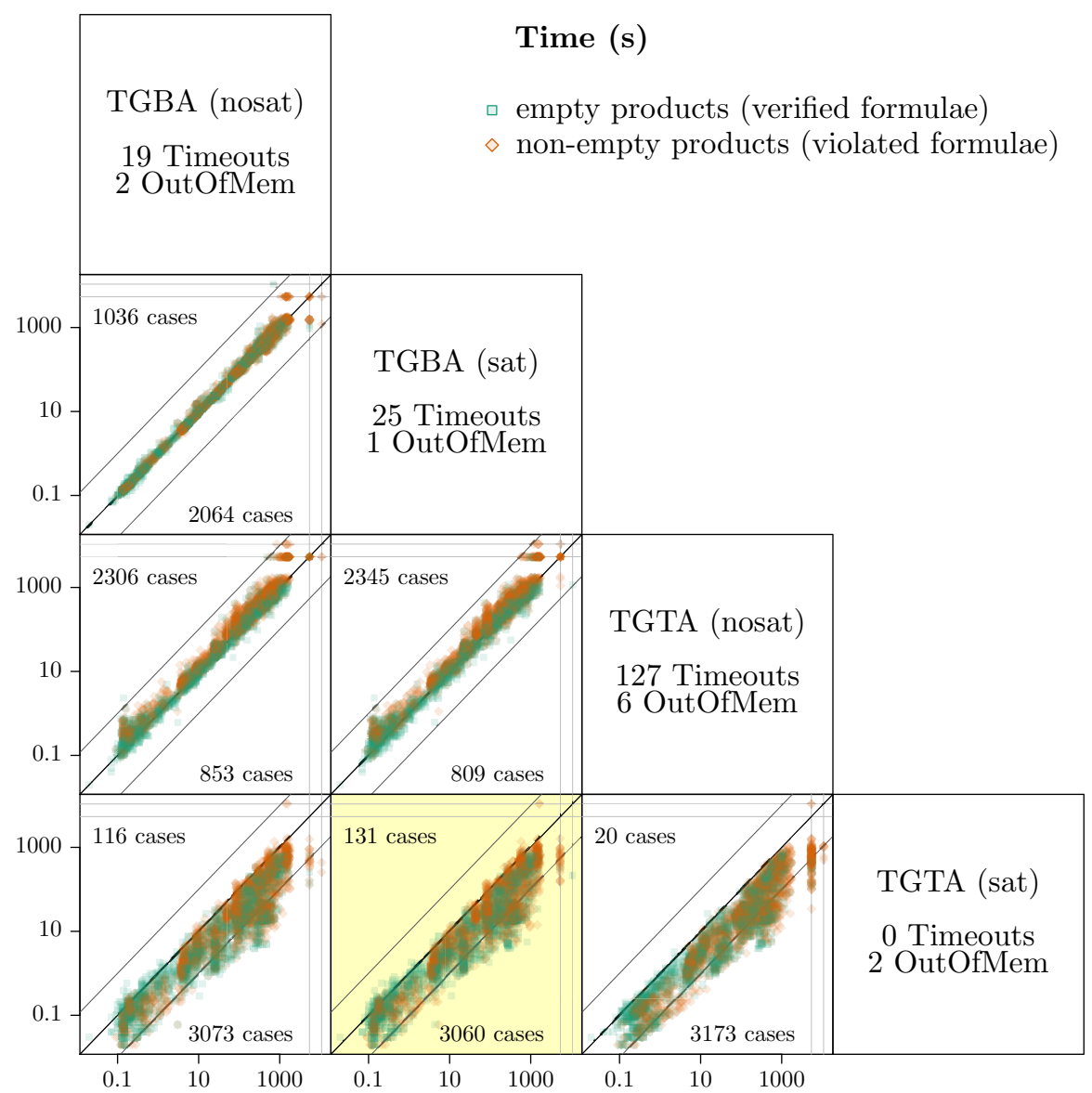

Fig. 3. Time-comparison of the TGBA and TGTA approaches, with saturation enabled "(sat)" or disabled "(nosat)", on a set of 3199 pairs (model,formula). Timeouts and Out-of-memory errors are plotted on separate lines on the top or right edges of the scatter plots. Each plot also displays the number of cases that are above or below the main diagonal (including timeouts and out-of-memory errors), i.e., the number of (model,formula) for which one approach was better than the other. Additional diagonals show the location of $\times 10$ and /10 ratios. Points are plotted with transparency to better highlight dense areas, and lessen the importance of outliers.

diagonal is in favor of the approach displayed in the top). Axes use a logarithmic scale. The colors distinguish violated formulae (non-empty product) from verified formulae (empty products). In order to show the influence of the saturation technique, we also ran the TGBA and TGTA approaches with saturation disabled. In our comparison matrix, the labels "(sat)" and "(nosat)" indicate whether saturation was enabled or not. Fig. 4 gives the memory view of this experiment. 


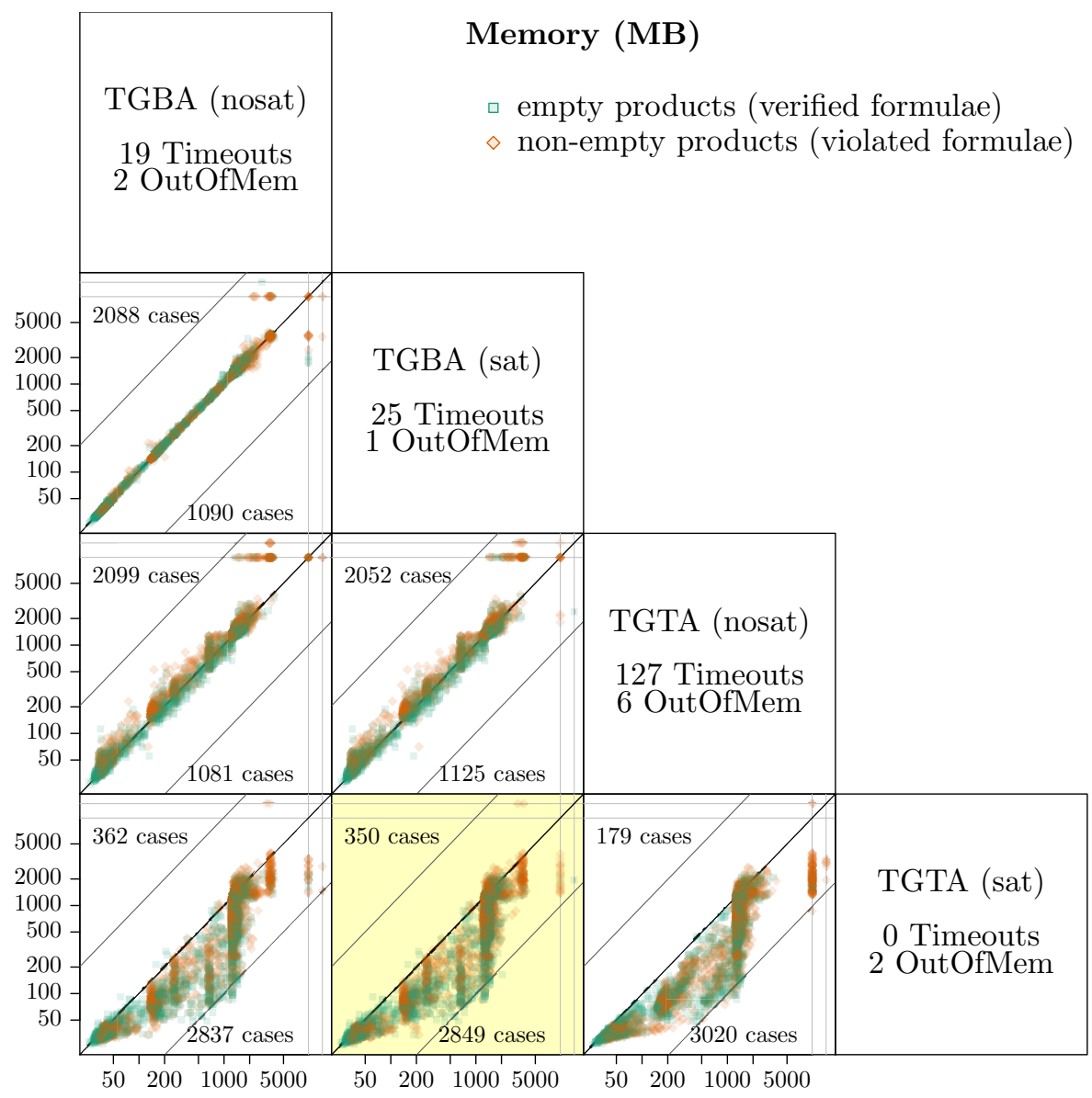

Fig. 4. Comparison of the memory-consumption of the TGBA and TGTA approaches, with or without saturation, on the same set of problems

As shown by the highlighted scatter plots in Fig. 3 and 4 the TGTA approach clearly outperforms the traditional TGBA-based scenario by one order of magnitude. This is due to the combination of two factors: saturation and exploration of stuttering.

The saturation technique does not significantly improve the model checking using TGBA (compare "TGBA (sat)" against "TGBA (nosat)" at the top of Fig. 3 and 4). In fact, the saturation technique is limited on the TGBA approach, because in the transition relation of Def.5 5 each conjunction must consult the variable $q$ representing the state of the TGBA, therefore $q$ impacts the supports and the reordering of clusters evaluated by the saturation. This situation is different in the case of TGTA approach, where the $T_{0}$ term of the transition-relation of the product (equation (1)) does not involve the state $q$ of the TGTA: here, saturation strongly improve performances (compare "TGTA (sat)" against "TGTA (nosat)"). 
Overall the improvement to this symbolic technique was only made possible because the TGTA representation makes it easy to process the stuttering behaviors separately from the rest. These stuttering transitions represent a large part of the models transitions, as shown by the stuttering-ratios of Table 1 . Using these stuttering-ratios, we can estimate in our Benchmark the importance of the term $T_{\emptyset}$ compared to $T_{*}$ in equation (1).

\section{Conclusion}

Testing automata [10] are a way to improve the explicit model checking approach when verifying stutter-invariant properties, but they had not been used for symbolic model checking. In this paper, we gave the first symbolic approach using testing automata, with generalized acceptance (TGTA), and compare it to a more classical symbolic approach (using TGBA).

On our benchmark, using TGTA, we were able to gain one order of magnitude over the TGBA-based approach.

We have shown that fixpoints over the transition relation of a product between a Kripke structure and a TGTA can benefit from the saturation technique, especially because part of their expression is only dependent on the model, and can be evaluated without consulting the transition relation of the property automaton. The improvement was possible only because TGTA makes it possible to process stuttering behaviors specifically, in a way that helps the saturation technique.

In future work, we plan to evaluate the use of TGTA in the context of hybrid approaches, mixing both explicit and symbolic approaches [18, 6].

\section{References}

1. Ben Salem, A.-E., Duret-Lutz, A., Kordon, F.: Model checking using generalized testing automata. In: Jensen, K., van der Aalst, W.M., Ajmone Marsan, M., Franceschinis, G., Kleijn, J., Kristensen, L.M. (eds.) ToPNoC VI. LNCS, vol. 7400, pp. 94-122. Springer, Heidelberg (2012)

2. Blom, S., van de Pol, J., Weber, M.: LTSMIN: Distributed and symbolic reachability. In: Touili, T., Cook, B., Jackson, P. (eds.) CAV 2010. LNCS, vol. 6174, pp. 354-359. Springer, Heidelberg (2010)

3. Burch, J.R., Clarke, E.M., McMillan, K.L., Dill, D.L., Hwang, L.: Symbolic model checking: $10^{20}$ states and beyond. In: Proc. of the Fifth Annual IEEE Symposium on Logic in Computer Science, pp. 1-33. IEEE Computer Society Press (1990)

4. Ciardo, G., Marmorstein, R., Siminiceanu, R.: Saturation unbound. In: Garavel, H., Hatcliff, J. (eds.) TACAS 2003. LNCS, vol. 2619, pp. 379-393. Springer, Heidelberg (2003)

5. Ciardo, G., Yu, A.J.: Saturation-based symbolic reachability analysis using conjunctive and disjunctive partitioning. In: Borrione, D., Paul, W. (eds.) CHARME 2005. LNCS, vol. 3725, pp. 146-161. Springer, Heidelberg (2005)

6. Duret-Lutz, A., Klai, K., Poitrenaud, D., Thierry-Mieg, Y.: Self-loop aggregation product - A new hybrid approach to on-the-fly LTL model checking. In: Bultan, T., Hsiung, P.-A. (eds.) ATVA 2011. LNCS, vol. 6996, pp. 336-350. Springer, Heidelberg (2011)

7. Duret-Lutz, A., Poitrenaud, D.: SPOT: an extensible model checking library using transitionbased generalized Büchi automata. In: Proc. of MASCOTS 2004, pp. 76-83. IEEE Computer Society Press (2004) 
8. Etessami, K.: Stutter-invariant languages, $\omega$-automata, and temporal logic. In: Halbwachs, N., Peled, D.A. (eds.) CAV 1999. LNCS, vol. 1633, pp. 236-248. Springer, Heidelberg (1999)

9. Fisler, K., Fraer, R., Kanhi, G., Vardi, M.Y., Yang, Z.: Is there a best symbolic cycle-detection algorithm? In: Margaria, T., Yi, W. (eds.) TACAS 2001. LNCS, vol. 2031, pp. 420-434. Springer, Heidelberg (2001)

10. Geldenhuys, J., Hansen, H.: Larger automata and less work for LTL model checking. In: Valmari, A. (ed.) SPIN 2006. LNCS, vol. 3925, pp. 53-70. Springer, Heidelberg (2006)

11. Giannakopoulou, D., Lerda, F.: From states to transitions: Improving translation of LTL formulæ to Büchi automata. In: Peled, D.A., Vardi, M.Y. (eds.) FORTE 2002. LNCS, vol. 2529, pp. 308-326. Springer, Heidelberg (2002)

12. Hamez, A., Thierry-Mieg, Y., Kordon, F.: Hierarchical set decision diagrams and automatic saturation. In: van Hee, K.M., Valk, R. (eds.) PETRI NETS 2008. LNCS, vol. 5062, pp. 211-230. Springer, Heidelberg (2008)

13. Hansen, H., Penczek, W., Valmari, A.: Stuttering-insensitive automata for on-the-fly detection of livelock properties. In: Proc. of FMICS 2002, ENTCS, vol. 66(2) (2002)

14. Kesten, Y., Pnueli, A., Raviv, L.-O.: Algorithmic verification of linear temporal logic specifications. In: Larsen, K.G., Skyum, S., Winskel, G. (eds.) ICALP 1998. LNCS, vol. 1443, pp. 1-16. Springer, Heidelberg (1998)

15. Pelánek, R.: BEEM: Benchmarks for explicit model checkers. In: Bošnački, D., Edelkamp, S. (eds.) SPIN 2007. LNCS, vol. 4595, pp. 263-267. Springer, Heidelberg (2007)

16. Peled, D., Wilke, T.: Stutter-invariant temporal properties are expressible without the nexttime operator. Information Processing Letters 63(5), 243-246 (1995)

17. Rozier, K.Y., Vardi, M.Y.: A multi-encoding approach for LTL symbolic satisfiability checking. In: Butler, M., Schulte, W. (eds.) FM 2011. LNCS, vol. 6664, pp. 417-431. Springer, Heidelberg (2011)

18. Sebastiani, R., Tonetta, S., Vardi, M.Y.: Symbolic systems, explicit properties: On hybrid approaches for LTL symbolic model checking. In: Etessami, K., Rajamani, S.K. (eds.) CAV 2005. LNCS, vol. 3576, pp. 350-363. Springer, Heidelberg (2005)

19. Somenzi, F., Ravi, K., Bloem, R.: Analysis of symbolic SCC hull algorithms. In: Aagaard, M.D., O'Leary, J.W. (eds.) FMCAD 2002. LNCS, vol. 2517, pp. 88-105. Springer, Heidelberg (2002)

20. Thierry-Mieg, Y., Poitrenaud, D., Hamez, A., Kordon, F.: Hierarchical set decision diagrams and regular models. In: Kowalewski, S., Philippou, A. (eds.) TACAS 2009. LNCS, vol. 5505, pp. 1-15. Springer, Heidelberg (2009)

21. Vardi, M.Y.: An automata-theoretic approach to linear temporal logic. In: Moller, F., Birtwistle, G. (eds.) Logics for Concurrency. LNCS, vol. 1043, pp. 238-266. Springer, Heidelberg (1996) 